\title{
Prevalence of Sarcopenia Was Higher in Women Than in Men: A Cross-Sectional Study From a Rural Area in Eastern China
}

\section{Yichen Yang}

First Affiliated Hospital Zhejiang University

Qin Zhang ( $\square$ zhangqin1978@zju.edu.cn )

First Affiliated Hospital Zhejiang University

Jing Chen

First Affiliated Hospital Zhejiang University

Caihong He

First Affiliated Hospital Zhejiang University

\section{Danfeng Deng}

First Affiliated Hospital Zhejiang University

Wenwen Lu

First Affiliated Hospital Zhejiang University

\section{Yuming Wang}

First Affiliated Hospital Zhejiang University

\section{Research Article}

Keywords: sarcopenia, rural health, women health, eastern China

Posted Date: November 15th, 2021

DOl: https://doi.org/10.21203/rs.3.rs-982218/v1

License: (c) (i) This work is licensed under a Creative Commons Attribution 4.0 International License. Read Full License 


\section{Abstract \\ Purpose}

There were limited studies specifically evaluating whether the difference of the prevalence of sarcopenia exists in men and women in older adults from rural areas in China. The aim of this study was to compare the prevalence of sarcopenia between men and women in a rural area in eastern China and to explore the underlying causes.

\section{Methods}

This study included 1,105 participants aged 60 89 years. Muscle mass was measured by bio-electrical impedance analysis. Hand grip strength was measured by Jamar Hydraulic Hand Dynamometer. Sarcopenia was diagnosed according to the Asian Working Group for Sarcopenia-2019 Consensus. Data were analyzed using a log-binomial and linear regression.

\section{Findings:}

The prevalence of sarcopenia was $21.7 \%$ in women and $12.9 \%$ in men among the study cohort. After adjusting for age, education level, number of diseases, smoking, drinking, and eating habits, the prevalence of sarcopenia was $151 \%$ times higher in women than in men $(P R=1.51,95 \% \mathrm{Cl}=1.01-2.26$, $\mathrm{P}<0.05)$.

\section{Conclusions}

The prevalence of sarcopenia in elderly women in this rural area of eastern China is higher than in men, suggesting that women in rural areas in China seems more vulnerable for sarcopenia, thus early screening and prevention need to be provided for them to eliminate such gender disparity in health.

\section{Introduction}

Sarcopenia is an aging related syndrome characterized by progressive loss of skeletal muscle mass and strength, which would lead to disability, decline of quality of life and even death in older adults.[1] Despite difference in populations, worldwide, the overall prevalence of sarcopenia in the elderly people over 60 years is about $10 \%$.[2] Sarcopenia is associated with increased risk of fall, fracture, prolonged hospital stays and increased mortality in older adults. These adverse health outcomes also increased the burden of family and social medical care.[3] The population aging process is serious in rural areas of China,[4] where the population has lower education level, less access to quality healthcare,[5] lower incomes and poor health condition[6] compared to those living in urban areas. Epidemiology studies reported different prevalence of sarcopenia in men and women, with majority reporting higher prevalence in men.[7] 
However, few studies have specifically evaluated whether the difference of the prevalence of sarcopenia exists in men and women in older adults from rural areas in China. Although women have longer life expectancy than men,[8] they have higher disease incidence rate and disability level, and studies have shown that the health status of elderly women is worse than that of old men.[9, 10] As women living in rural areas are disadvantaged group in terms of education, employment, and income level, it is important to identify whether they are vulnerable population for sarcopenia so that tailored intervention could be provided.

The aim of this study was to compare the prevalence of sarcopenia between men and women in rural areas of Doumen Town, Shaoxing City, Zhejiang Province, China, and to explore the related factors for this difference.

\section{Methods}

\section{Study Participants}

The data are from a national key research and development program (No.2018YFC2000301), which is a national multi-center study assessing the characteristics of health status changes in the process of aging in Chinese population. From May to August 2019, we recruited people aged 60-89 from several villages in Doumen Town, Shaoxing City, Zhejiang Province, China. The inclusion criteria were: 1. Local residents with relatively stable work or residence; 2 . No acute or chronic infectious diseases; 3 . No progressive fatal diseases; 4 . No serious mental illness; 5 . No history of alcohol or drug abuse. The following situation was excluded: Those who had any physical or mental function problems and could not complete the survey. We confirm that all methods were performed in accordance with the 2019 consensus update on sarcopenia diagnosis and treatment from Asian Working Group for Sarcopenia.

\section{Muscle Mass Measurement}

Appendicular skeletal muscle mass (ASM) was measured by bio-electrical impedance analysis (BIA) (BCA-2A, Tsinghua Tongfang). Acknowledging the difficulty of measuring muscle mass in community settings, the Asian Working Group for Sarcopenia-2014 (AWGS 2014) supported ASM measurement using bioelectrical impedance analysis (BIA).[11] AWGS 2019 continues this view. Before the test, subjects were asked to be fasting for 4 hours and abstain from alcohol for 8 hours. The subjects were wearing light clothes, standing barefoot on the instrument, legs slightly opened, hands held electrodes, arms were away from the trunk. Appendicular muscle mass $(\mathrm{kg}) /$ height $^{2}\left(\mathrm{~m}^{2}\right)$ was used to reflect the muscle mass $\left(A S M=k g / m^{2}\right)$.

\section{Grip Strength Measurement}

Jamar Hydraulic Hand Dynamometer (5030J1, Sammons Preston, Inc., Bolingbrook, III., USA) was used in this study. The standard positionings were sitting with 90 elbow flexion recommended by AWGS 2019. 
Took the maximum reading of at least 2 trials using either both hands or the dominant hand in a maximum-effort isometric contraction.[7]

\section{Demographic and health information}

All participants completed a survey questionnaire and trained researchers got answers from participants or their families through face-to-face interviews. The survey collected information on education level, smoking, drinking, chronic diseases, physical exercise and eating habits. Educational level was divided into three categories: illiterate, primary school, secondary school and above. Smoking status was divided into three categories: never smoking, current smoking and ever smoking. Drinking status was divided into three categories: never drinking, current drinking and ever drinking.

Chronic diseases were assessed by asking participants whether they had any of the following diseases or conditions, including: high blood pressure, diabetes, heart diseases, stroke or cerebrovascular disease, respiratory diseases (bronchitis, COPD and asthma), pulmonary tuberculosis, cataract, chronic kidney disease, cancer, gastrointestinal diseases, Parkinson's disease, falls, arthritis, dementia, metabolic disorder, cervical and lumbar diseases. According to the disease reported, number of chronic diseases was divided into four groups: no disease, one chronic disease, two chronic diseases, more than two chronic diseases.

Physical exercise was assessed by asking whether taking regular excises (seldom or often). If reporting yes, the types and time per week were assessed. Eating habits were assessed by asking the frequency of consumption of meat, fish, eggs, milk, beans, vegetables and fruits. For each aspect of eating habit, the reported frequency had four categories: do not eat, eat occasionally, often eat and eat every day.

\section{Statistical Analysis}

Continuous variables in accordance with normal distribution were expressed as means (standard deviation), and the comparison between the two groups was conducted by two independent sample ttest. The categorical variables were expressed by the number of cases and percentages, and the comparison between groups was performed by $\chi 2$ test. Prevalence ratio (PR) and $95 \%$ confidence interval (Cl) were obtained by log binomial regression. In order to determine the related factors of the difference in the prevalence of sarcopenia between men and women, multivariate log binomial regression analysis was performed. $\mathrm{P}<0.05$ was determined as statistically significant. All the analysis were performed in STATA 14.

\section{Results}

We recruited 1,105 older adults, aged 60-89 years, including 558 men and 547 women. The mean age were $72.4(0.31)$ years old in men, and 70.94 (0.30) years in women. Figure 1 shows the screening process of sarcopenia in the study cohort.

\section{Participants Characteristics}


The education level of women was significantly lower than that of men $(P<0.001)$, women smoked less $(P<0.001)$ and drank less $(P<0.001)$ than men, women had more chronic diseases than men $(P=0.001)$. Compared to men, women ate less meat $(P<0.001)$, fish $(P<0.001)$, eggs $(P<0.001)$ and milk $(P=0.004)$. Women and men's eating habits of vegetables $(P=0.154)$ and fruits $(P=0.343)$ were similar; physical exercise habits were similar too $(P=0.622)$ (Table 1$)$.

\section{Prevalence of Sarcopenia, LGS and LMM}

The overall prevalence of sarcopenia in this rural population was $17.29 \%$ (191/1105), with the prevalence in women $(21.72 \%)$ was significantly higher than that in men (12.93\%). The prevalence of low grip strength (LGS) and low muscle mass (LMM) was 46.50\% (259/557) and 21.72\% (121/557) in men, while $53.47 \%(293 / 548)$ and $27.01 \%$ (148/548) in women respectively (Table 2).

\section{Associations between sex and sarcopenia, LGS and LMM}

We then conducted log binomial regression analysis to evaluate the difference of prevalence of sarcopenia, LGS and LMM between men and women. Results showed that, compared to men, the prevalence of sarcopenia was $168 \%$ times higher $(P R=1.68,95 \% \mathrm{Cl}=1.29-2.20, \mathrm{P}<0.001)$, the prevalence of LGS was $115 \%$ times higher $(P R=1.15,95 \% \mathrm{Cl}=1.02-1.29, \mathrm{P}<0.05)$ and the prevalence LMM was $124 \%$ times higher $(P R=1.24,95 \% \mathrm{Cl}=1.01-1.53, \mathrm{P}<0.05)$ in women. After adjusting for age, education level and number of diseases, the associations for sarcopenia and LMM remained statistically significant. For example, the multivariate regression model (Model 1) showed that women were more likely to have sarcopenia $(P R=1.61,95 \% \mathrm{Cl}=1.19-2.18, \mathrm{P}<0.05)$ and $\mathrm{LMM}(\mathrm{PR}=1.27,95 \% \mathrm{Cl}=1.00-1.61$, $P<0.05)$. However, there was no difference in $L G S$ between women and men $(P R=1.03,95 \% \mathrm{Cl}=0.91$ $1.17, P>0.05)$.

On the basis of Model 1, we further adjusted for smoking, drinking and eating habits (consumption of meat, egg, fish and milk) that differed between men and women thus constructed the second multivariable regression model (Model 2). The results showed that the prevalence of sarcopenia was still higher in women than in men $(P R=1.51,95 \% \mathrm{Cl}=1.01-2.26, \mathrm{P}<0.05)$, but differences in $\mathrm{LGS}(\mathrm{PR}=0.97$, $95 \% \mathrm{Cl}=0.81-1.16, \mathrm{P}>0.05)$ and $\mathrm{LMM}(\mathrm{PR}=1.31,95 \% \mathrm{Cl}=0.96-1.79, \mathrm{P}>0.05)$ disappeared (Table 3$)$.

\section{Discussion}

Using the AWGS 2019 sarcopenia diagnostic criteria, we found that the prevalence of sarcopenia was $168 \%$ times higher in women (prevalence $21.72 \%$ ) than in men $(12.93 \%$ ) among older adults from a rural area in eastern China. Adjusting for age, education level and number of diseases only slightly reduced the effect size for the prevalence of sarcopenia and low muscle mass but the association between sex and low grip strength became nonsignificant, suggesting that the difference in the prevalence of sarcopenia was mainly driven by the higher prevalence of low muscle mass in women rather than low grip strength. After further adjustment for smoking, drinking, number of chronic diseases and eating habits, the prevalence of sarcopenia in women was still significant higher. 
Our study found that elderly women were more likely to have sarcopenia than men in a rural area in eastern China. However, the review of epidemiology studies conducted in Asian countries reported that the prevalence of sarcopenia was more predominant in men than in women (5.1\%-21.0\% in men vs 4.1\%-16.3\% in women).[7] The different source of population might explain the different prevalence across studies. While there were limited epidemiological studies assessing prevalence of sarcopenia that specifically restricted to older adults from rural areas in China, it was reported that older adults from rural area in China were much more likely to have worse health outcomes such as malnutrition and frailty.[6] Compared to men, older women from rural areas were more disadvantaged as they generally had lower education level and lower income than men.[6] It has been reported that the prevalence of disability was higher in women than in men in older adults from rural China.[12] The prevalence of sarcopenia increases gradually with age, $[13,14]$ and is associated with increased risk of falls, lower quality of life and many chronic diseases such as diabetes, cardiovascular disease, heart failure, renal insufficiency, cancer, cognitive impairment, Parkinson's syndrome and depression, and even the prognosis of chronic diseases. [15-17] To maintain better health status and eliminate gender inequality in health, it is important to provide early screening and effective interventions for sarcopenia in older female adults in the rural community of China.

Adjusting for sociodemographic characteristics, number of diseases and lifestyle behavior factors in the multivariable model reduced the association, suggesting that the difference in prevalence of sarcopenia was partially explained by these factors. But the remained significant higher prevalence of sarcopenia in women suggested that there were other factors other than the above factors strongly driving this difference. While smoking was shown to be a risk factor for sarcopenia,[18, 19] and the proportion of people smoking was much higher in men than in women, we still observed significant higher prevalence of sarcopenia in women after adjusting for smoking.

In our study, we only used simple questions to measure diet and physical exercise so that we were unable to quantify nutrition intake from diet and physical exercise level from different domains. Thus, our results should be interpreted with caution that the mediating effects of diet and physical exercise on the association between sex and sarcopenia could not be excluded. Adequate intake and absorption of energy and protein are important for older adults to maintain muscle health.[20-22] Studies have shown nutritional deficiency increased the risk of sarcopenia. $[23,24]$

Protein, vitamin D and calcium supplementation can improve skeletal and muscle health in postmenopausal women.[25] In our study cohort, we found that women reported less frequency intake of eggs, milk, fish and meats, which are the major source of protein intake from foods. Future studies using food frequency questionnaire or dietary recall to quantify the specific nutrient intake are needed to assess whether and to what extent poor diet quality explained the higher prevalence of sarcopenia in women.

In addition, elderly people who lack physical exercise are more likely to experience skeletal muscle mass loss and muscle strength reduction, thus leading to increased risk of developing sarcopenia.[24, 26] In our study cohort, around $80 \%$ reported no regular exercise in both men and women. Since the majority older 
adults from rural areas in China were traditional Chinese farmers, and we did not measure physical exercise from domestic and farm works, it was possible that men involved more in physical-demanding farm works or other works than women, which may benefit them with better muscle health.

There were some other factors associated with the development of sarcopenia that were not assessed in the current study. Age-related changes in the endocrine system and hormone levels play an important role in the pathogenesis of sarcopenia. For example, testosterone could increase muscle mass and enhance muscle function, and reduction in testosterone level is involved in the development of sarcopenia.[27] It has been observed that androgen supplements play an important role in promoting muscle strength and increasing muscle mass. Recently, a study on 94 elderly people with normal thyroid function found that higher concentration of free triiodothyronine (FT3) in the normal range was positively correlated with the muscle mass and muscle function of the elderly.[28] Other factors related to sarcopenia include motor neuron degeneration,[29] genetic factors,[30] inflammatory factor[31, 32] and insulin resistance.[33, 34] Gender difference has been found in the process of aging. $[35,36]$ Whether the above factors explained the observed difference in the prevalence of sarcopenia between men and women needs further researches.

The strengths of our study include that we used the latest diagnostic criteria for sarcopenia from Asian Working Group for Sarcopenia (AWGS 2019) that combined low grip strength and low muscle mass. To date, there were few studies evaluating sarcopenia using the updated criteria. Secondly, we specifically focused on older adults from rural areas in Zhejiang Province with large sample size. There were several limitations need to be acknowledged of the current study. First of all, we used convenient sample that were recruited in Doumen Town, which might not be representative of the general rural older residence in Zhejiang Province, China. In addition, we did not use detailed validated questionnaire to quantify nutrition intake from diet and physical activity, which limited us to comprehensively assess their effects on the observed associations. Lastly, for chronic diseases, we used the count of self-reported diseases, which did not capture the severity of diseases.

\section{Conclusions}

In conclusion, according to the AWGS 2019 diagnostic criteria, the prevalence of sarcopenia was higher in elderly women than that in men in a rural area of eastern China. The finding suggests that women in rural areas in China seems more vulnerable for sarcopenia, thus early screening and prevention need to be provided for them to eliminate such gender disparity in health. Adjusting for sociodemographic, chronic diseases and lifestyle behaviors reduced the associations but the difference remained significant, suggesting that there were other factors strongly driving the difference. To provide more helpful information for health policy makers and to develop more tailored intervention programs, further studies are needed to explore the possible reasons for such difference.

\section{Declarations}


The protocol and procedure were approved by the ethics committee of the First Affiliated Hospital of Zhejiang University. Written informed consent was obtained from individual. According to the declaration of Helsinki and relevant clinical trial management norms in China, when the subject is illiterate with full civil capacity, informed consent shall be given in the presence of the witness. All the subjects of the study had full civil capacity. The staff of Doumen community acted as witnesses. Under their witness, the participates understood the relevant contents of the study and orally agreed to participate in the study. After that, they pressed their fingerprints on the informed consent form. In addition, some illiterate elderly people could sign their names and they did it.

\section{Consent for publication}

Not applicable.

\section{Availability of data and materials}

Researchers are willing to share the original data of the study. The datasets used and analysed during the current study are available from the corresponding author on reasonable request.

\section{Competing interests}

We declare that we have no conflicts of interest.

\section{Funding}

This study was supported by 2 national key research and development programs of China (No. 2018YFC2000301, Funder: Qin Zhang; No.2018YFC2001405, Funder: Qin Zhang) and 2 medical health science and technology projects of Zhejiang Provincial Health Commission (No. 202038269, Funder: Danfeng Deng; No.2021430262, Funder: Yuming Wang).

\section{Authors' contributions}

Conceptualization: Jing Chen; Methodology: Jing Chen, Qin Zhang; Formal analysis and investigation: Yichen Yang, Caihong He, Yuming Wang, Wenwen Lu, Danfeng Deng; Writing-original draft preparation: Yichen Yang; Writing-review and editing: Yichen Yang, Qin Zhang, Jing Chen; Funding acquisition: Qin Zhang, Yuming Wang, Danfeng Deng; Resources: Qin Zhang; Supervision: Qin Zhang.

\section{Acknowledgements}

We appreciated the community staff of Doumen Town for their testimony and other contributions to the smooth progress of the study.

\section{References}


1. Dennison EM, Sayer AA, Cooper C: Epidemiology of sarcopenia and insight into possible therapeutic targets. Nature reviews Rheumatology 2017, 13(6):340-347.

2. Shafiee G, Keshtkar A, Soltani A, Ahadi Z, Larijani B, Heshmat R: Prevalence of sarcopenia in the world: a systematic review and meta- analysis of general population studies. Journal of diabetes and metabolic disorders 2017, 16:21.

3. Beaudart C, Zaaria M, Pasleau F, Reginster JY, Bruyère O: Health Outcomes of Sarcopenia: A Systematic Review and Meta-Analysis. PloS one 2017, 12(1):e0169548.

4. Yu P, Liu X, Wang J: Geriatric medicine in China: The past, present, and future. Aging medicine (Milton (NSW)) 2018, 1(1):46-49.

5. Dong X, Zhang H, Wang F, Liu X, Yang K, Tu R, Wei M, Wang L, Mao Z, Zhang G et al: Epidemiology and prevalence of hyperuricemia among men and women in Chinese rural population: The Henan Rural Cohort Study. Modern rheumatology 2020, 30(5):910-920.

6. Dai BZ, Zhou LL, Mei YJ: Old age security in rural China: there is a long way to go. Chinese medical journal 2013, 126(22):4348-4353.

7. Chen LK, Woo J, Assantachai P, Auyeung TW, Chou MY, lijima K, Jang HC, Kang L, Kim M, Kim S et al: Asian Working Group for Sarcopenia: 2019 Consensus Update on Sarcopenia Diagnosis and Treatment. Journal of the American Medical Directors Association 2020, 21(3):300-307.e302.

8. Fang EF, Scheibye-Knudsen M, Jahn HJ, Li J, Ling L, Guo H, Zhu X, Preedy V, Lu H, Bohr VA et al: A research agenda for aging in China in the 21st century. Ageing research reviews 2015, 24(Pt B):197205.

9. Burton-Jeangros C, Zimmermann-Sloutskis D: Life satisfaction trajectories of elderly women living in Switzerland: an age-period-cohort analysis. Ageing \& Society 2016, 36(1):106-132.

10. Liu M, He Y, Jiang B, Wu L, Wang J, Yang S, Wang Y, Li X: Association between reproductive variables and metabolic syndrome in chinese community elderly women. Arch Gerontol Geriatr 2016, 63:7884.

11. Chen LK, Liu LK, Woo J, Assantachai P, Auyeung TW, Bahyah KS, Chou MY, Chen LY, Hsu PS, Krairit $O$ et al: Sarcopenia in Asia: consensus report of the Asian Working Group for Sarcopenia. Journal of the American Medical Directors Association 2014, 15(2):95-101.

12. Stewart Williams J, Norström F, Ng N: Disability and ageing in China and India - decomposing the effects of gender and residence. Results from the WHO study on global AGEing and adult health (SAGE). BMC geriatrics 2017, 17(1):197.

13. Martinez BP, Batista AK, Gomes IB, Olivieri FM, Camelier FW, Camelier AA: Frequency of sarcopenia and associated factors among hospitalized elderly patients. BMC musculoskeletal disorders 2015, 16:108.

14. Yu R, Wong M, Leung J, Lee J, Auyeung TW, Woo J: Incidence, reversibility, risk factors and the protective effect of high body mass index against sarcopenia in community-dwelling older Chinese adults. Geriatrics \& gerontology international 2014, 14 Suppl 1:15-28. 
15. Chen LK, Lee WJ, Peng LN, Liu LK, Arai H, Akishita M: Recent Advances in Sarcopenia Research in Asia: 2016 Update From the Asian Working Group for Sarcopenia. Journal of the American Medical Directors Association 2016, 17(8):767.e761-767.

16. Hsu YH, Liang CK, Chou MY, Liao MC, Lin YT, Chen LK, Lo YK: Association of cognitive impairment, depressive symptoms and sarcopenia among healthy older men in the veterans retirement community in southern Taiwan: a cross-sectional study. Geriatrics \& gerontology international 2014, 14 Suppl 1:102-108.

17. Srikanthan P, Karlamangla AS: Relative muscle mass is inversely associated with insulin resistance and prediabetes. Findings from the third National Health and Nutrition Examination Survey. The Journal of clinical endocrinology and metabolism 2011, 96(9):2898-2903.

18. Rom O, Kaisari S, Aizenbud D, Reznick AZ: Sarcopenia and smoking: a possible cellular model of cigarette smoke effects on muscle protein breakdown. Annals of the New York Academy of Sciences 2012, 1259:47-53.

19. Steffl M, Bohannon RW, Petr M, Kohlikova E, Holmerova I: Relation between cigarette smoking and sarcopenia: meta-analysis. Physiological research 2015, 64(3):419-426.

20. Komar B, Schwingshackl L, Hoffmann G: Effects of leucine-rich protein supplements on anthropometric parameter and muscle strength in the elderly: a systematic review and meta-analysis. The journal of nutrition, health \& aging 2015, 19(4):437-446.

21. Liao CD, Tsauo JY, Wu YT, Cheng CP, Chen HC, Huang YC, Chen HC, Liou TH: Effects of protein supplementation combined with resistance exercise on body composition and physical function in older adults: a systematic review and meta-analysis. The American journal of clinical nutrition 2017, 106(4):1078-1091.

22. Lord C, Chaput JP, Aubertin-Leheudre M, Labonté M, Dionne IJ: Dietary animal protein intake: association with muscle mass index in older women. The journal of nutrition, health \& aging 2007, 11(5):383-387.

23. Molnár A, Jónásné Sztruhár I, Csontos Á A, Ferencz C, Várbíró S, Székács B: Special nutrition intervention is required for muscle protective efficacy of physical exercise in elderly people at highest risk of sarcopenia. Physiology internationa/ 2016, 103(3):368-376.

24. Naseeb MA, Volpe SL: Protein and exercise in the prevention of sarcopenia and aging. Nutrition research (New York, NY) 2017, 40:1-20.

25. Rizzoli R, Stevenson JC, Bauer JM, van Loon LJ, Walrand S, Kanis JA, Cooper C, Brandi ML, DiezPerez A, Reginster JY: The role of dietary protein and vitamin D in maintaining musculoskeletal health in postmenopausal women: a consensus statement from the European Society for Clinical and Economic Aspects of Osteoporosis and Osteoarthritis (ESCEO). Maturitas 2014, 79(1):122-132.

26. Burton LA, Sumukadas D: Optimal management of sarcopenia. Clinical interventions in aging 2010, 5:217-228.

27. Herbst KL, Bhasin S: Testosterone action on skeletal muscle. Current opinion in clinical nutrition and metabolic care 2004, 7(3):271-277. 
28. Sheng Y, Ma D, Zhou Q, Wang L, Sun M, Wang S, Qi H, Liu J, Ding G, Duan Y: Association of thyroid function with sarcopenia in elderly Chinese euthyroid subjects. Aging clinical and experimental research 2019, 31(8):1113-1120.

29. Drey M, Krieger B, Sieber CC, Bauer JM, Hettwer S, Bertsch T: Motoneuron loss is associated with sarcopenia. Journal of the American Medical Directors Association 2014, 15(6):435-439.

30. Tan LJ, Liu SL, Lei SF, Papasian CJ, Deng HW: Molecular genetic studies of gene identification for sarcopenia. Human genetics 2012, 131(1):1-31.

31. Frost RA, Lang $\mathrm{CH}$ : Protein kinase B/Akt: a nexus of growth factor and cytokine signaling in determining muscle mass. Journal of applied physiology (Bethesda, Md: 1985) 2007, 103(1):378387.

32. Patel HP, Al-Shanti N, Davies LC, Barton SJ, Grounds MD, Tellam RL, Stewart CE, Cooper C, Sayer AA: Lean mass, muscle strength and gene expression in community dwelling older men: findings from the Hertfordshire Sarcopenia Study (HSS). Calcif Tissue Int 2014, 95(4):308-316.

33. Kwon SS, Lee SG, Lee YH, Lim JB, Kim JH: Homeostasis model assessment of insulin resistance in a general adult population in Korea: additive association of sarcopenia and obesity with insulin resistance. Clinical endocrinology 2017, 86(1):44-51.

34. Moon SS: Low skeletal muscle mass is associated with insulin resistance, diabetes, and metabolic syndrome in the Korean population: the Korea National Health and Nutrition Examination Survey (KNHANES) 2009-2010. Endocrine journal 2014, 61(1):61-70.

35. Marais GAB, Gaillard JM, Vieira C, Plotton I, Sanlaville D, Gueyffier F, Lemaitre JF: Sex gap in aging and longevity: can sex chromosomes play a role? Biology of sex differences 2018, 9(1):33.

36. Thomas N, Gurvich C, Kulkarni J: Sex Differences in Aging and Associated Biomarkers. Advances in experimental medicine and biology 2019, 1178:57-76.

\section{Tables}


Table 1. Participants Characteristics.

\begin{tabular}{|c|c|c|c|}
\hline & Men & Women & $P$ value \\
\hline & $N=557$ & $N=548$ & \\
\hline Age (years), mean (SD) & $72.4(0.31)$ & 70.94(0.30) & $\varangle 0.001$ \\
\hline Education level, n (\%) & & & $₫ 0.001$ \\
\hline illiterate & 164(29.71) & $406(74.50)$ & \\
\hline Primary & $306(55.43)$ & 125(22.94) & \\
\hline Secondary and above & $82(14.86)$ & $14(2.57)$ & \\
\hline Number of diseases, n (\%) & & & 0.001 \\
\hline No & 147(26.39) & 126(22.99) & \\
\hline 1 & $259(46.50)$ & 212(38.69) & \\
\hline 2 & 101(18.13) & 135(24.64) & \\
\hline More than 2 & $50(8.98)$ & 75(13.69) & \\
\hline Smoking, n (\%) & & & $\bowtie 0.001$ \\
\hline Never & $269(48.47)$ & $540(99.08)$ & \\
\hline current & 189(34.05) & $4(0.73)$ & \\
\hline Ever & $97(17.48)$ & $1(0.18)$ & \\
\hline Drinking, n (\%) & & & $₫ 0.001$ \\
\hline Never & 203(36.51) & 442(80.95) & \\
\hline current & 292(52.52) & $93(17.03)$ & \\
\hline Ever & $61(10.97)$ & $11(2.01)$ & \\
\hline Physical exercise, n (\%) & & & 0.622 \\
\hline seldom & 437(78.88) & 424(77.66) & \\
\hline often & 117(21.12) & $122(22.34)$ & \\
\hline Meat, n (\%) & & & $₫ 0.001$ \\
\hline no & 49(8.81) & 202(36.93) & \\
\hline occasionally & 193(34.71) & 205(37.48) & \\
\hline often & $269(48.38)$ & $124(22.67)$ & \\
\hline everyday & $45(8.09)$ & $16(2.93)$ & \\
\hline
\end{tabular}




\begin{tabular}{|c|c|c|c|}
\hline no & $34(6.12)$ & $187(34.25)$ & \\
\hline occasionally & 168(30.22) & $178(32.60)$ & \\
\hline often & $337(60.61)$ & 177(32.42) & \\
\hline everyday & $17(3.06)$ & $4(0.73)$ & \\
\hline Eggs, n (\%) & & & $\nabla 0.001$ \\
\hline no & $65(11.69)$ & $223(40.77)$ & \\
\hline occasionally & $279(50.18)$ & $223(40.77)$ & \\
\hline often & 189(33.99) & $88(16.09)$ & \\
\hline everyday & $23(4.14)$ & 13(2.38) & \\
\hline Milk, n (\%) & & & 0.004 \\
\hline no & $278(50.00)$ & $332(60.81)$ & \\
\hline occasionally & $154(27.70)$ & $114(20.88)$ & \\
\hline often & $81(14.57)$ & $69(12.64)$ & \\
\hline everyday & $43(7.73)$ & $31(5.68)$ & \\
\hline Vegetables, n (\%) & & & 0.154 \\
\hline no & $4(0.72)$ & $3(0.55)$ & \\
\hline occasionally & $5(0.90)$ & $0(0.00)$ & \\
\hline often & $53(9.53)$ & $57(10.44)$ & \\
\hline everyday & 494(88.85) & 486(89.01) & \\
\hline Fruits, n (\%) & & & 0.343 \\
\hline no & $56(10.09)$ & $50(9.17)$ & \\
\hline occasionally & $325(58.56)$ & $306(56.15)$ & \\
\hline often & $151(27.21)$ & $154(28.26)$ & \\
\hline everyday & $23(4.14)$ & $35(6.42)$ & \\
\hline
\end{tabular}

SD: standard deviation. 
Table2. Sex Differences in Sarcopenia, LGS and LMM

\begin{tabular}{|c|c|c|c|}
\hline & $\operatorname{Men}(n=557)$ & Women $(n=548)$ & $P$ \\
\hline \multicolumn{3}{|c|}{ Sarcopenia, n (\%) } & $\bowtie 0.001$ \\
\hline Yes & 72(12.93) & 119(21.72) & \\
\hline No & 485(87.07) & $429(78.28)$ & \\
\hline \multicolumn{3}{|c|}{ LGS, n (\%) } & 0.021 \\
\hline Yes & $259(46.50)$ & 293(53.47) & \\
\hline No & 298(53.50) & $255(46.53)$ & \\
\hline \multicolumn{2}{|c|}{ LMM, n (\%) } & 0.041 & \\
\hline Yes & 121(21.72) & 148(27.01) & \\
\hline No & $436(78.28)$ & $400(72.99)$ & \\
\hline
\end{tabular}

LGS low grip strength, LMM low muscle mass.

Table 3. Associations between sex and sarcopenia, LGS and LMM

$\begin{array}{lll}\text { Univariable } & \text { Multivariable model }{ }^{*} & \text { Multivariablemodel* }^{*} \\ \text { PR }(95 \% \mathrm{Cl}) & \text { PR }(95 \% \mathrm{Cl}) & \text { PR }(95 \% \mathrm{Cl})\end{array}$

Associations between sex and sarcopenia

Men 1 (reference) 1 (reference) 1 (reference)

Women $\quad 1.68(1.29-2.20) * * \quad 1.61(1.19-2.18)^{*} \quad 1.51(1.01-2.26)$ *

Associations between sex and LGS

$\begin{array}{llll}\text { Men } & 1 \text { (reference) } & 1 \text { (reference) } & 1 \text { (reference) } \\ \text { Women } & \mathbf{1 . 1 5}(\mathbf{1 . 0 2 - 1 . 2 9 ) *} & 1.03(0.91-1.17) & 0.97(0.81-1.16)\end{array}$

Associations between sex and LMM

$\begin{array}{llll}\text { Men } & 1 \text { (reference) } & 1 \text { (reference) } & 1 \text { (reference) } \\ \text { Women } & \mathbf{1 . 2 4}(\mathbf{1 . 0 1 - 1 . 5 3 ) *} & \mathbf{1 . 2 7 ( 1 . 0 0 - 1 . 6 1 ) *} & 1.31(0.96-1.79)\end{array}$

Data in bold indicate statistical significance; ${ }^{*} p<0.05 ; * \star p<0.001$. PR: prevalence ratio; Cl: confidence interval. LGS low grip strength, LMM low muscle mass.

${ }^{¥}$ Adjusted for age, education level and number of diseases. * Adjusted for age, education level, number of diseases, smoking, drinking, and eating habits of meat, egg, fish and milk. 
Figures

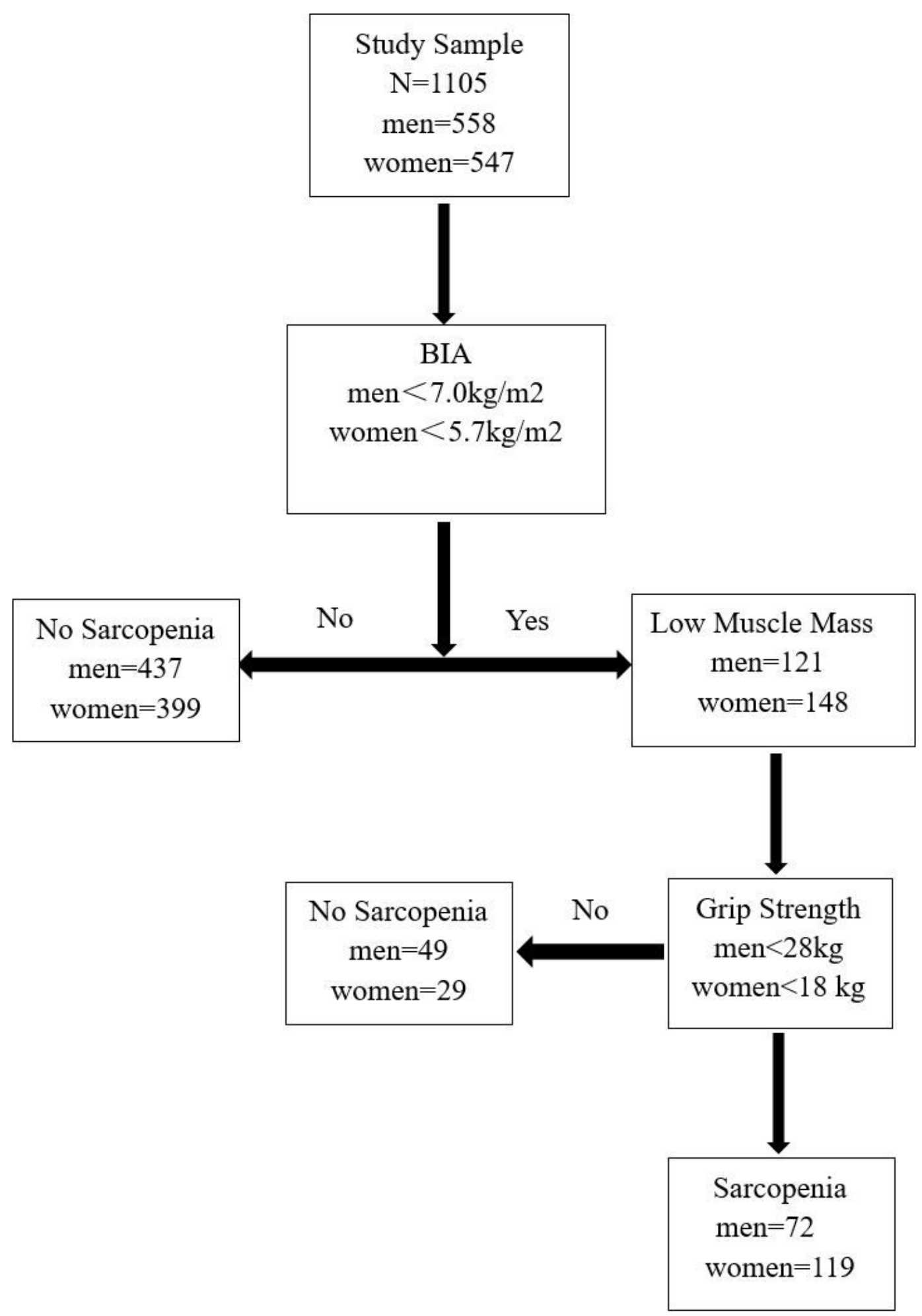

Figure 1

Application of AWGS 2019 algorithm for the case finding of sarcopenia. 